\title{
A model for orientation effects in electron-transfer reactions
}

\author{
Paul Siders, a) Robert J. Cave, and R. A. Marcus \\ Noyes Laboratory of Chemical Physics, California Institute of Technology, ${ }^{\text {b) }}$ Pasadena, California 91125
}

(Received 18 June 1984; accepted 14 August 1984)

\begin{abstract}
A method for solving the single-particle Schrödinger equation with an oblate spheroidal potential of finite depth is presented. The wave functions are then used to calculate the matrix element $T_{\mathrm{BA}}$ which appears in theories of nonadiabatic electron transfer. The results illustrate the effects of mutual orientation and separation of the two centers on $T_{\mathrm{BA}}$. Trends in these results are discussed in terms of geometrical and nodal structure effects. Analytical expressions related to $T_{\mathrm{BA}}$ for states of spherical wells are presented and used to analyze the nodal structure effects for $T_{\mathrm{BA}}$ for the spheroidal wells.
\end{abstract}

\section{INTRODUCTION}

The mutual orientation of the donor and acceptor in an electron transfer reaction may have observable effects on the electron transfer rate in certain systems. For example, the primary photoinduced electron transfer in photosynthetic reaction centers may be influenced by the orientation of the reactants. In plant photosystem II the electron acceptor is probably a pheophytin ${ }^{1,2}$ and the donor may be a substituted chlorophyll $a$ monomer. ${ }^{2,3}$ Both of these molecules are large and nonspherical suggesting that there may be one or more preferred orientations for electron transfer. Another biologically important electron transfer, that between hemes in cytochromes, may also depend on the mutual orientation of the porphyrin rings of the hemes. ${ }^{4}$

Orientation effects are beginning to be examined experimentally in model systems. For example, electron transfer between cofacial porphyrins has been studied and was observed to be rapid. ${ }^{5,6}$ Systems involving porphyrins held in other orientations are under study. ${ }^{7}$ In these systems the electron transfer is between sites that are chemically linked. When the pi-type orbitals at the donor and acceptor sites are largely electronically independent, the electron transfers may be treated using the usual outer-sphere formalism. It is with systems such as these in mind that we have set out to develop a model theoretical system within which to examine the nature and magnitude of orientation effects on electrontransfer rates.

The rate constant for nonadiabatic electron transfer between reactants $A$ and $B$ at fixed separation and orientation has been examined within the Golden Rule formalism, e.g., ${ }^{8-10}$

$$
k=\frac{2 \pi}{\hbar}\left|T_{\mathrm{BA}}\right|^{2} \mathbf{F C} .
$$

The Franck-Condon sum (here denoted FC) has been discussed in detail elsewhere, for example. ${ }^{10-12}$ In this paper we consider the dependence, within the theoretical model described below, of the electronic matrix element $T_{\mathrm{BA}}$ on the mutual orientation and separation distance of $A$ and $B$.

The matrix element $T_{\mathrm{BA}}$ depends on the electronic

\footnotetext{
a) Present address: Radiation Laboratory, University of Notre Dame, Notre Dame, Indiana 46556.

b) Contribution No. 7040
}

wave functions localized on sites A and B. An isolated electronic site A or B (at infinite separation say) is modeled in this paper as an oblate spheroid, and the potential for the electron is set equal to a negative constant inside the well and zero outside. It may be recalled that an oblate spheroid can be obtained by rotating an ellipse about its minor axis.

The volume of the spheroidal potential well is supposed to enclose the carbon skeleton of an aromatic system. The circle of revolution generated by the major axis when the spheroid is rotated about its minor axis is imagined to lie in the plane of the carbon skeleton. Other models have similarly exploited the delocalized character of the pi electrons in aromatic systems. In the free electron molecular orbital model, ${ }^{13}$ e.g., the electron is free to move in one dimension on a ring or intersecting rings, but has zero probability density off of the ring. In another model introduced by Schmidt ${ }^{14}$ and developed by Platt ${ }^{15}$ to calculate electron densities and electronic spectra of aromatics, the electron is free to move in a plane in a region bounded by infinite potential walls. In contrast, in the present paper the electronic wave function is three dimensional and is not confined to a well, because the potential used is finite. The wave functions therefore have long range tails which are important in describing electron transfer.

The present model yields a predominately exponential dependence of the rate on separation distance, a dependence used or found in various experimental studies. ${ }^{16}$ The molecular basis of this model may actually be an exchange mechanism involving orbitals of adjacent molecules or atoms. ${ }^{17}$

There have been previous discussions of orientation effects in the context of the tunneling of trapped electrons in glassy matrices. Rice et al..$^{18}$ considered orientation effects in a qualitative way, and concluded that orientation dependence in the electron tunneling rate would be equivalent to a reduced concentration of electron acceptors, and thereby reduce the tunneling relative to an analogous system with no orientation dependence. Brocklehurst ${ }^{19}$ examined the orientation dependence of the overlap of electronic wave functions for spherically symmetric sites. He considered both hydrogenic and spherical-well potentials. The electronic matrix element was assumed by Brocklehurst to be proportional to the overlap of the wave functions, an approximation which we consider using states of spherical wells in Appendix $\mathrm{B}$. He concluded that the orientation effect on the electron-transfer rate constant can be as large as $10^{3}$. Dok- 
torov et $a l^{20}$ considered an angular factor, $\cos ^{n} \Theta$, in the unimolecular rate constant for electron tunneling between spherical sites. For $n \leqslant 4$, the effect of this angular dependence was to reduce the overall rate constant. The present paper considers sites that are asymmetric and therefore geometrically orientable and which presumably better represent the aromatic systems toward which this study is aimed.

Spectral properties of porphyrin compounds have been examined by numerous workers using semiempirical electronic structure methods. ${ }^{21} \mathrm{Ab}$ initio techniques have also been used to examine porphyrin electronic structure. ${ }^{22,23}$ Calculations on diporphyrin systems and their low-lying charge transfer states have been recently reported. ${ }^{24}$ Electronic structure techniques have been used to study orientation and distance effects for a model transition metal redox pair $^{25}$ as well as for face-to-face porphyrins at small separation distances using both semiempirical and ab initio methods. ${ }^{26,27}$ For porphyrin electron transfers $a b$ initio calculations of the electronic matrix element are extremely lengthy. Moreover, ab initio techniques which employ Gaussian basis functions are better suited to describe wave functions inside molecules than to depict the long-range tails of the wave functions. While our model is significantly less detailed than that on which these wave functions are based, the present aim is to include the general features of the problem. In fact, it is the simplicity of the model which facilitates the calculations presented here.

The paper is organized as follows. In Sec. II the model for the potential and the wave functions used are briefly described, the calculation of the electron transfer matrix element is outlined, and results from calculations of the matrix element are presented. In Sec. III a more detailed description of the calculation of the single-site wave functions is given. The results for the electron transfer matrix element calculations are discussed in Sec. IV. Concluding remarks are given in Sec. V. The relation between the Golden Rule rate expression and the matrix element is given in Appendix A. Expressions for the matrix element for states of spherical wells are derived in Appendix B. Applications to molecules of experimental interest will be presented in a subsequent article.

\section{WAVE FUNCTIONS AND RESULTS}

\section{A. The model}

The model involves the interaction of two sites, labeled A and B (e.g., molecules or electronically isolated chromophores). The single-site wave functions are taken to be oneelectron wave functions, i.e., only the transferable electron is considered explicitly. The potential in which the electron moves is modeled as an oblate spheroidal well. A cross section of the potential is sketched in Fig. 1. The potential is independent of $\varphi$, the angle of rotation about the $z$ axis. The cross section is an ellipse having semimajor axis $a$, semiminor axis $b$, and eccentricity $e \equiv \sqrt{1-b^{2} / a^{2}}$. The potential $V$ is zero outside the well and has a constant negative value inside. Actually, there will also be a Coulombic term when the molecule is charged, ${ }^{28}$ but it is assumed, for the present, that in a medium with some polarity this contribution is small relative to the values of $V_{0}$ used below.

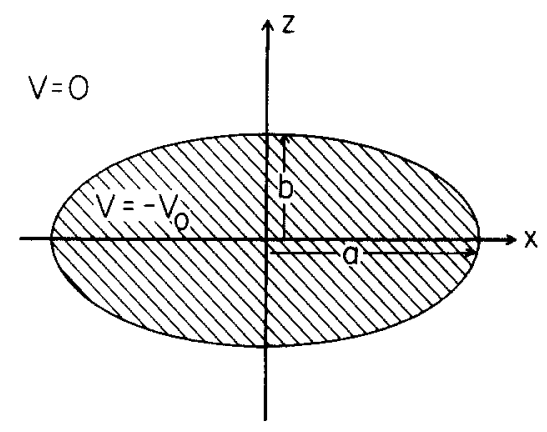

FIG. 1. Potential well for a single site. There is cylindrical symmetry about the $z$ axis.

It is convenient to use oblate spheroidal coordinates $(\xi, \eta, \varphi)$, defined by

$$
\begin{aligned}
& x=\frac{1}{2} d\left[\left(1+\xi^{2}\right)\left(1-\eta^{2}\right)\right]^{1 / 2} \cos \varphi, \\
& y=\frac{1}{2} d\left[\left(1+\xi^{2}\right)\left(1-\eta^{2}\right)\right]^{1 / 2} \sin \varphi, \\
& z=\frac{1}{2} d \xi \eta,
\end{aligned}
$$

where $0 \leqslant \xi,-1 \leqslant \eta \leqslant 1,0 \leqslant \varphi \leqslant 2 \pi$. The scale factor $d$ has been chosen so that the surface of the potential well is described by the single radial-like coordinate $\xi$. With $d=2 \sqrt{a^{2}-b^{2}}, V$ is defined as

$$
V=\left\{\begin{array}{l}
-V_{0} ; \quad \xi \leqslant \xi_{0} \equiv 2 b / d \\
0 ; \quad \xi>\xi_{0}
\end{array} .\right.
$$

Contours of the coordinate system are presented in Fig. 2. (The angular coordinate $\varphi$, not shown, is defined as for spherical coordinates.) The surface $\xi=0$ is a disk of diameter $d$. The surface $\eta=0$ is the $x y$ plane minus this disk.

Spherical coordinates $r$ and $\theta$ are given in terms of oblate spheroidal coordinates:

$$
\begin{aligned}
& r=\frac{d}{2}\left(1+\xi^{2}-\eta^{2}\right)^{1 / 2}, \\
& \cos \theta=\xi \eta\left(1+\xi^{2}-\eta^{2}\right)^{-1 / 2}
\end{aligned}
$$

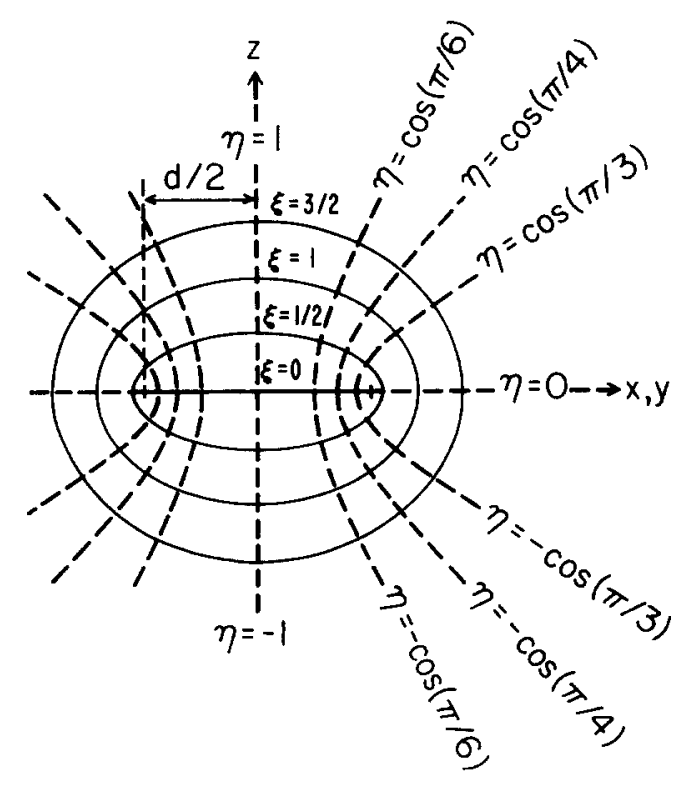

FIG. 2. Oblate-spheroidal coordinate system. Contours of constant $\xi$ are indicated by solid lines. The dashed lines are contours of constant $\eta$. The contours of constant $\eta$ on the right are for $\varphi=0$, on the left for $\varphi=\pi$. 
It is clear that the oblate spheroidal coordinates become spherical coordinates at asymptotically large distances from the potential, in the sense that $\xi \rightarrow 2 r / d$ and $\eta \rightarrow \cos \theta$ as $r \rightarrow \infty$.

The single-site wave functions sought are bound-state solutions to the Schrödinger equation with the potential of Eq. (3). The Schrödinger equation may be written as a pair of Helmholtz's equations, one satisfied inside the well and one outside, i.e.,

$$
\left(\nabla^{2}+k^{2}\right) \Psi=0
$$

with $k^{2}=2 m_{e}\left(E+V_{0}\right) / \hbar^{2}$ when $\xi \leqslant \xi_{0}$ and $k^{2}=2 m_{e} E / \hbar^{2}$ when $\xi>\xi_{0}, m_{e}$ being the electronic mass. The value of $V_{0}$ affects the eigenvalue $E$ and hence controls the rate of decay of the wave function with distance and ultimately the decay of the thermal matrix element.

In Sec. III it is shown that the wave function may be written as

$$
\begin{aligned}
& \Psi_{m t u}= \begin{cases}\Psi_{m t u}^{i}(\xi, \eta, \varphi) \equiv A_{m t u} & \sum_{r=0}^{\infty} C_{n}^{i} \psi_{m n}^{i} ; \quad \xi \leqslant \xi_{0}, \\
\Psi_{m t u}^{o}(\xi, \eta, \varphi) \equiv A_{m t u} & \sum_{r=0}^{\infty} C_{n}^{o} \psi_{m n}^{o} ; \quad \xi \geqslant \xi_{0},\end{cases} \\
& n=2 r+m+s .
\end{aligned}
$$

The value of $s$ determines the parity of the wave function relative to the $x y$ plane of the potential, $\Psi_{m i u}$ being even when $s=0$ and odd when $s=1$. The superscript $i$ or $o$ denotes the wave function inside or outside the well, respectively.

The functions $\psi_{m n}^{i}$ and $\psi_{m n}^{o}$ are solutions of the Schrödinger equation in oblate spheroidal coordinates when the potential is a constant over all space and is equal to its inner or outer value, respectively. Quantization in the case of a finite depth well is accomplished by requiring continuity of the wave function $\Psi_{m t u}$ and its derivative at the boundary, i.e., at $\xi=\xi_{0}$.

The functions $\psi_{m n}^{i, o}$ are separable in oblate spheroidal coordinates and may be written as

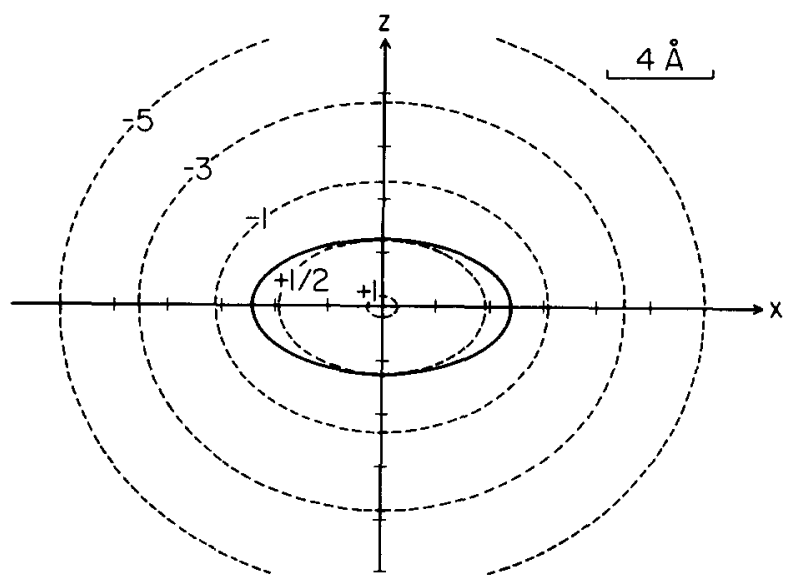

FIG. 3. Contours of $\Psi$ for a state with $(m, t, u)=(0,0,1), V_{0}=10 \mathrm{eV}$, $E=-7.98 \mathrm{eV}, a=4.85 \AA, b=2.55 \AA$. The heavy line is the well boundary. The contours are labeled with values of $\log _{10}|\Psi|$. This state is referred to later as a $(0, \sigma)$ state.

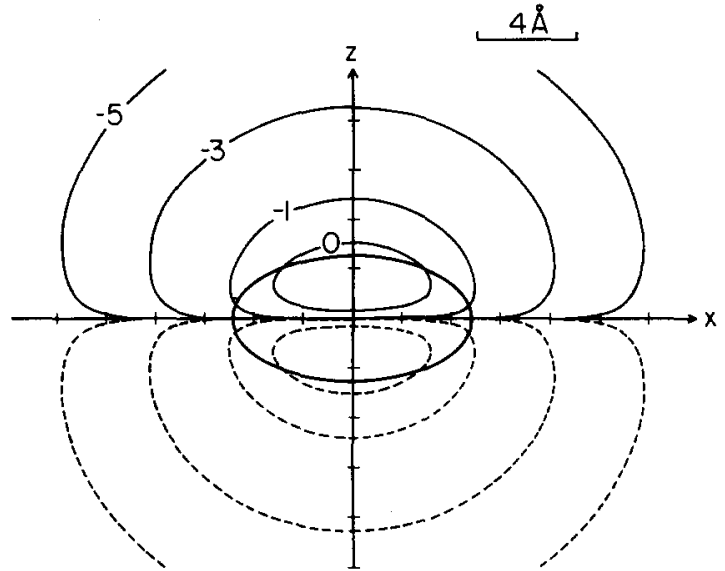

FIG. 4. Contours of $\Psi$ for a state with $(m, t, u)=(0,1,1) . a, b$, and $V_{0}$ are as in Fig. 3. $E=-4.70 \mathrm{eV}$. The heavy line is the well boundary. The contours are labeled with values of $\log _{10}|\Psi|$. Dashed contours indicate $\Psi<0$. Solid contours are for $\Psi>0$. This state is referred to later as a $(0, \pi)$ state.

$\psi_{m n}^{i}(\xi, \eta, \varphi)=R_{m n}^{i}\left(\xi ; k_{i}^{2}\right) S_{m n}^{i}\left(\eta ; k_{i}^{2}\right) \Phi_{m}(\varphi), \quad \xi \leqslant \xi_{0}$,

$\psi_{m n}^{o}(\xi, \eta, \varphi)=R_{m n}^{o}\left(\xi ; k_{o}^{2}\right) S_{m n}^{o}\left(\eta ; k_{o}^{2}\right) \Phi_{m}(\varphi), \quad \xi \geqslant \xi_{0}$.

$\Phi_{m}(\varphi)$ may be written as a linear combination of $\sin m \varphi$ and $\cos m \varphi$ and the number of nodes in $\Phi_{m}(\varphi)$ is equal to $2 m$. The index $n$ has been chosen to have the possible values $n=m, m+1, m+2 \ldots$.

The quantum numbers $t$ and $u$ in Eq. (6) will be described as follows: $t$ is the number of nodal lines in the two dimensional $\xi \eta$ subspace and $u$ orders states of equal $t$ by energy $(u=1,2, \ldots)$. At fixed $V_{0}, a$, and $b$, a wave function can usually be specified using $m, t$, and $u$. (Near an avoided crossing the nodal lines become increasingly complicated, however. When the nodal structure is not too distinct one could simply use $m$ and a parameter which orders states of the given $m$ by energy.)

Contour plots of wave functions for several states having $m=0$ are shown in Figs. 3-5. Energy levels ${ }^{29}$ for several states are shown in Fig. 6 as a function of eccentricity at constant volume for the infinite potential case [Fig. 6(a)] and

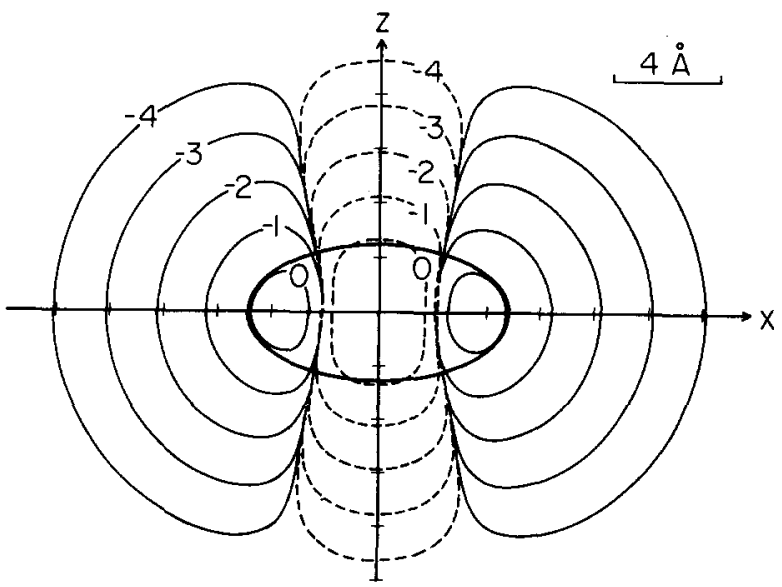

FIG. 5. Contours of $\Psi$ for a state with $(m, t, u)=(0,1,2) . a, b$, and $V_{0}$ are as in Fig. 3, $E=-4.44 \mathrm{eV}$. The various lines are as in Fig. 4. 

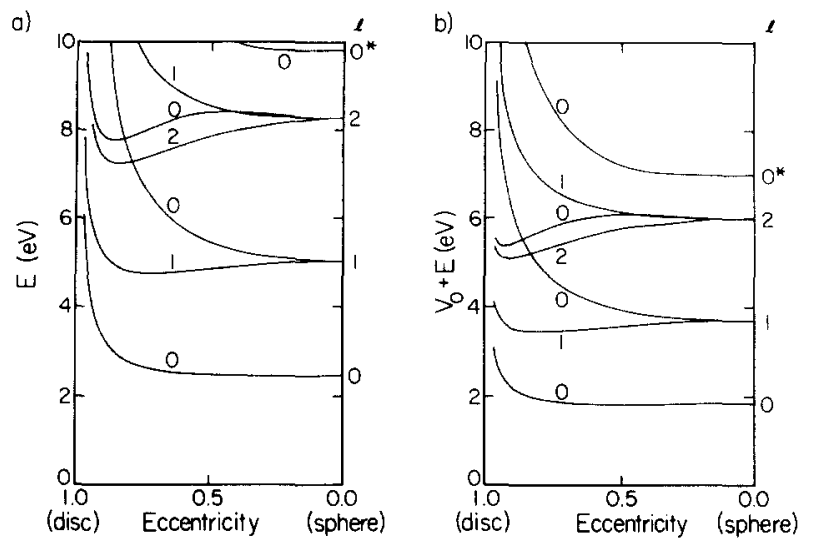

FIG. 6. Energies relative to the bottom of the potential well vs eccentricity. In both (a) and (b) the wells have a constant volume of $251.25 \AA^{3}$. The effective spherical radius $R_{\text {eff }}\left[\equiv\left(a^{2} b\right)^{1 / 3}\right]$ for these results is $3.9145 \AA$. Energy levels are labeled with $l$ on the right-hand side of the figure and $m$ above individual curves, where $l$ and $m$ are the total angular momentum and its $z$ projection of the state of the spherical well to which a given spheroidal state correlates. The $m$ and $l$ quantum numbers of the states in Figs. 3-5 are $(m, l)=(0,0),(0,1)$, and $(0,2)$, respectively. An asterisk indicates the presence of a radial nodal surface in the spherical wave function. $V_{0}$ equals $\infty$ in (a) and $10 \mathrm{eV}$ in (b). In both cases only those spheroidal states which correlate to the four lowest distinct spherical energy eigenvalues are shown.

for $V_{0}=10 \mathrm{eV}$ [Fig. 6(b)]. Energy levels are shown as a function of $V_{0}$ in Fig. 7.

For calculations of $T_{\mathrm{BA}}$ most of the states we have studied have one nodal line in the $\xi \eta$ subspace (i.e., $t=1$ ) and $s=1$. Such wave functions provide the closest analog to $2 p \pi$ electron systems, $s=1$ being appropriate to $\pi$-like symmetry, since these functions are odd with respect to the $x y$ plane. For simplicity we will use the notation $(m, \pi)$ to denote a state with $t=1$ and $s=1$ for a given $m$ for the rest of the article, $\pi$ denoting odd symmetry with respect to reflection in the $x y$ plane. The $\pi$-like nature of the $(0, \pi)$ state is apparent in Fig. 4. For comparison with experimental systems designed to assess orientation and distance effects we note that a $(1, \pi)$ state has the same nodal structure as the HOMO in benzene. $A(4, \pi)$ state has the same nodal structure as the HOMO in porphine, as determined in ab initio calculations. ${ }^{22}$ To illustrate a particular geometrical effect we have

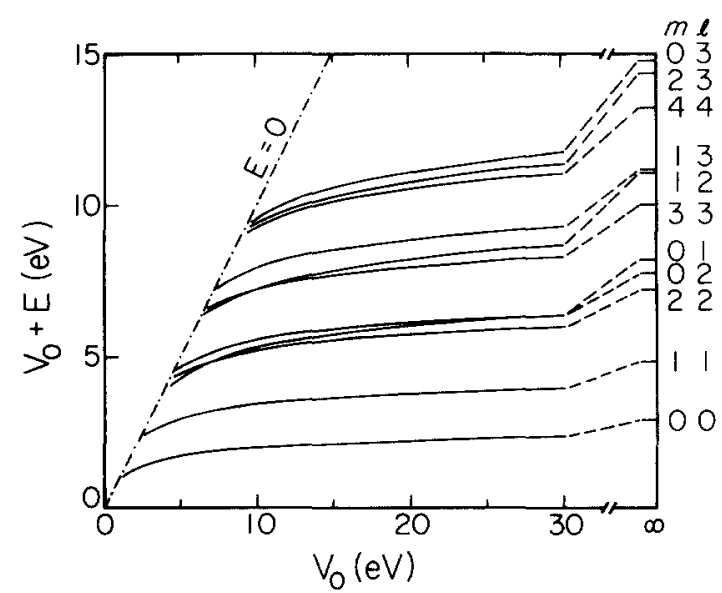

FIG. 7. Energies relative to the bottom of the well vs well depth. $a$ and $b$ as in Fig. 3, volume $=251.25 \AA^{3} . m$ and $l$ are indicated on the right-hand side of the figure and are defined as for Fig. 6. also given some results for states with zero nodes. We will refer to them as $(0, \sigma)$ states, since $m$ is zero and, like $\sigma$ states, they are even with respect to reflection in the $x y$ plane of the potential.

\section{B. Electron transfer between sites}

The system used to model electron transfer between a pair of molecules A and B consists of two wells (site A and site $B$ ), each of the type described previously, and one electron (the "transferable" electron). The rate constant for the electron transfer reaction

$$
\mathrm{A}+\mathrm{B} \rightarrow \mathrm{A}+\mathrm{B}
$$

is given by Eq. (1), using the Golden Rule and Condon approximations. That rate constant is for transfer between sites having specific and fixed mutual orientations and fixed relative separation distance. In order to use Eq. (1), nuclear coordinates and an associated set of vibrational states have been assumed to be present in the wells and in the intervening medium (along with solvent orientational states), but will not be dealt with explicitly in this paper. Recent reviews on this aspect of the electron transfer problem are given in Ref. 30.

The zeroth-order problem is that in which the two wells do not interact (e.g., the infinite-separation limit). The following two zeroth-order states are considered:

(1) The electronic state at site A, uninfluenced by site $B$ : The wave function for this state, denoted by $\Psi_{m u}^{\mathrm{A}}$, is given by Eq. (6), with the origin of the coordinates at the center of well $\mathrm{A}$ and with $\xi_{0}$ defining the boundary of well $\mathrm{A}$. The $V_{0}$ appearing in Eq. (3) and appropriate to site $\mathrm{A}$ is denoted by $V_{0}^{\mathrm{A}}$.

(2) The electronic state at site B, uninfluenced by site A, which has as its wave function $\Psi_{m^{\prime} t^{\prime} u^{\prime}}^{\mathrm{B}}$, given by Eq. (6), but centered now on site $\mathrm{B}$, and having $V_{0}=V_{0}^{\mathrm{B}}$.

The electronic matrix element $T_{\mathrm{BA}}$, described in Appendix A for the present model, is

$$
T_{\mathrm{BA}}=\left(H_{\mathrm{BA}}-S_{\mathrm{AB}} H_{\mathrm{AA}}\right) /\left(1-\left|S_{\mathrm{AB}}\right|^{2}\right),
$$

where

$$
\begin{aligned}
& H_{\mathrm{BA}}=-V_{0}^{\mathrm{B}} \int \Psi_{m^{\prime} t^{\prime} u^{\prime}}^{\mathrm{B}^{*}} \Psi_{m t u}^{\mathrm{A}} d \tau_{\mathrm{B}}, \\
& H_{\mathrm{AA}}=-V_{0}^{\mathrm{B}} \int \Psi_{m t u}^{\mathrm{A}} \Psi_{m t u}^{\mathrm{A}} d \tau_{\mathrm{B}}, \\
& S_{\mathrm{AB}}=\int \Psi_{m t u}^{\mathrm{A}^{*}} \Psi_{m t^{\prime} u^{\prime}}^{\mathrm{B}} d \tau .
\end{aligned}
$$

The integrals in Eq. (10a) are over well B, and that in Eq $(10 b)$ is over all space.

\section{Results of calculations of the electron transfer matrix element}

Calculations of the electron transfer matrix element were performed with various eigenstates of each of the two separated wells with specific fixed mutual orientations. The states and orientations chosen illustrate some general effects of the shape of the potential well and orientation on the matrix element.

Mutual orientations of the two wells are defined using 


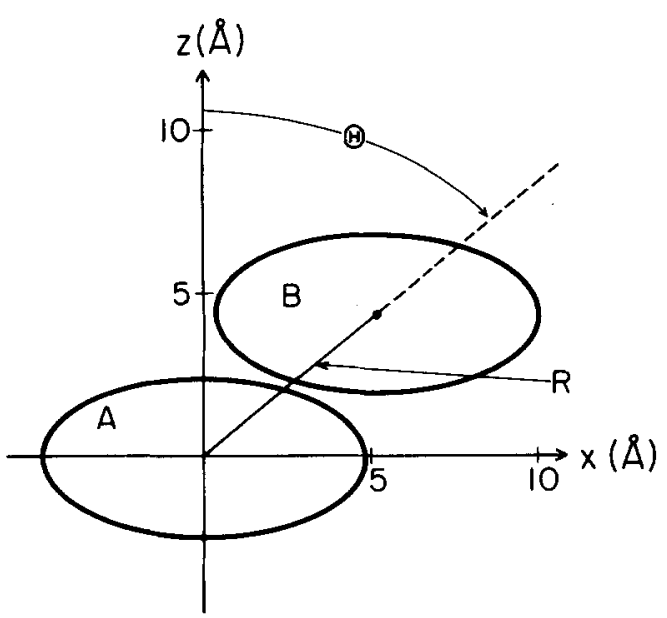

FIG. 8. Coordinate system used to specify the mutual orientation of well $A$ and well $B$. The $x$ axes of the wells are assumed parallel and lie in the plane of the figure in all geometries. $\Theta=0^{\circ}$ corresponds to the $z$ axes of the wells being superimposed.

the coordinate system in Fig. 8. Well A was assumed fixed and well B was positioned at various values of $R$ and $\Theta$. In the calculations given here the $x$ axes of the wells are parallel, as are the $y$ axes. $\Theta=0^{\circ}$ corresponds to the wells being displaced along the $z$ axis, and so being in a "face-to-face" configuration. $\Theta=90^{\circ}$ corresponds to displacement along the $x$ axis, i.e., in an "end-to-end" arrangement.

The values of $a$ and $b$ used (apart from those in Fig. 6) were chosen as follows: $a$ was an estimate of the in-plane radius of porphine, and is the same $a$ as that used by Platt ${ }^{15}$ to treat porphine as a $2 a \times 2 a$ square using the Schmidt box model. The value of $b$ was chosen so that the average thickness of the well $(=4 b / 3)$ corresponded to the interplane

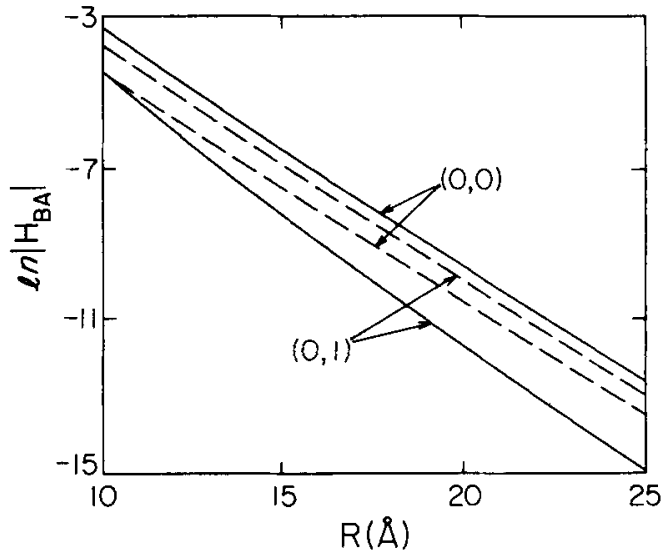

FIG. 9. $\ln \left|H_{\mathrm{BA}}\right|$ as a function of center-to-center separation for a pair of $(0, \sigma)[=(0,0)]$ states in each well and for a pair of $(0, \pi)[=(0,1)]$ states. $a$ and $b$ are as in Fig. 3. $E=-1.1525 \mathrm{eV} ; V_{0}$ is $2.5937 \mathrm{eV}$ for the $(0, \sigma)$ states and $5.6540 \mathrm{eV}$ for the $(0, \pi)$ states. Solid lines are for $\Theta=90^{\circ}$ and dashed lines for $\Theta=0^{\circ}$.

spacing in graphite, $3.4 \AA .^{31}$ Other values of $b$ are of course possible. The general features of the orientation dependence are not qualitatively affected by the choice of $b$.

$T_{\mathrm{BA}}$ and $H_{\mathrm{BA}}$ are compared for the wells at contact for various states and various angles $\Theta$ in Table $I$. It can be seen that typically $T_{\mathrm{BA}}$ and $H_{\mathrm{BA}}$ agree to within $5 \%$. The agreement becomes even better with increased separation. The calculation of $H_{\mathrm{BA}}$ is much less time consuming than $T_{\mathrm{BA}}$, and only values of $H_{\mathrm{BA}}$ are given in the rest of the article. The trends seen are unaffected.

Results as a function of distance and orientation are plotted in Figs. 9-11 for pairs of $(0, \sigma)$ and $(0, \pi)$ states. In Figs. 9-12 $V_{0}$ was chosen so that each state, independently of $m$ and of the parity, has an energy $E=-1.1525 \mathrm{eV}$. This

TABLE I. Comparison of $H_{\mathrm{BA}}$ and $T_{\mathrm{BA}}$ at selected angles $\Theta$ for potential wells at contact.,

\begin{tabular}{cccc}
\hline \hline State & $\Theta(\mathrm{deg})$ & $H_{\mathrm{BA}}(\mathrm{eV})$ & $H_{\mathrm{BA}} / T_{\mathrm{BA}}$ \\
\hline$(0, \sigma)$ & 0 & -0.263 & 1.000 \\
& 30 & -0.229 & 1.000 \\
& 45 & -0.182 & 1.006 \\
& 60 & -0.121 & 1.008 \\
$(0, \pi)$ & 90 & -0.045 & 1.004 \\
& 0 & 0.666 & 1.084 \\
& 30 & 0.474 & 1.092 \\
& 45 & 0.267 & 1.074 \\
$(2, \pi)$ & 60 & 0.077 & 1.025 \\
& 90 & -0.015 & 1.000 \\
& 0 & 0.481 & 1.007 \\
& 30 & 0.040 & 1.069 \\
& 45 & 0.109 & 1.029 \\
$(4, \pi)$ & 60 & 0.299 & 1.035 \\
& 90 & -0.062 & 1.002 \\
& 0 & 0.246 & 1.001 \\
& 30 & -0.079 & 1.012 \\
& 45 & 0.113 & 1.013 \\
& 60 & 0.279 & 1.019 \\
& 90 & -0.066 & 1.002 \\
\hline
\end{tabular}

s The wells have $a=4.85 \AA, b=2.55 \AA$, and $V_{0}$ such that $E=-1.1525 \mathrm{eV}$. For $(0, \sigma),(0, \pi),(2, \pi)$, and $(4, \pi)$, states, $V_{0}=2.5937,5.6540,10.6541$, and $17.3530 \mathrm{eV}$, respectively. In the worst case $H_{\mathrm{BA}}$ is converged to within $2 \%$ of the exact value. In general, the convergence is much better.

${ }^{\circ} \Phi_{m}(\varphi)=\cos m \varphi$. Similar agreement of $H_{\mathrm{BA}}$ and $T_{\mathrm{BA}}$ is seen for $\Phi_{m}(\varphi)=\sin m \varphi$ or any linear combination of $\sin m \varphi$ and $\cos m \varphi$. 


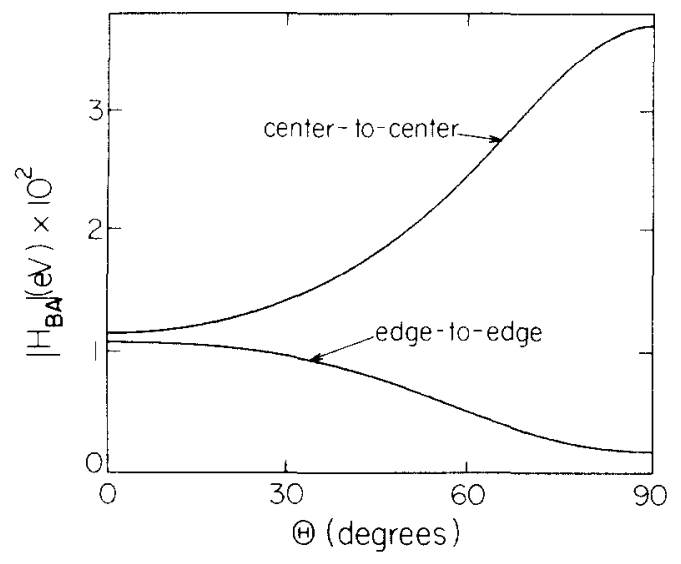

FIG. 10. $\left|H_{\mathrm{BA}}\right|$ as a function of $\Theta$ for a pair of $(0, \sigma)$ states. $a, b, V_{0}$, and $E$ are as in Fig. 9 for the $(0, \sigma)$ states. Shown are results for a constant center-tocenter distance of $10 \AA$ and for a constant edge-to-edge distance of $5 \AA$.

value of $E$ yields states which cause $\left|H_{\mathrm{BA}}\right|^{2}$ to have an (approximately) exponential decay whose slope of a $\ln \left|H_{\mathrm{BA}}\right|^{2} \mathrm{vs}$ $R$ plot is from 1.4 to 1.7 depending on the states involved. These are in the range of some experimental estimates for the decay of the electronic matrix element with distance for aromatic molecule-aromatic anion systems. ${ }^{16}$ [The rate in Ref. 16 (a) is proportional to $\exp (-2 \alpha R)$, where $2 \alpha$ is roughly 1.1 $\AA^{-1}$.]

In Table II $H_{\mathrm{BA}}$ is given as a function of distance for $\Theta=0^{\circ}$ and $90^{\circ}$ for the $(1, \pi),(2, \pi)$, and $(4, \pi)$ states. Each eigenvalue of states with $m>0$ is twofold degenerate. The functions $\cos m \varphi$ or $\sin m \varphi$ or any linear combination of them are eigenfunctions of the $\varphi$ portion of the Schrödinger equation and $H_{\mathrm{BA}}$ will in general depend on which of these functions is chosen, as well as on the relative orientation of the wells. In actual molecules of current interest, deviations from cylindrical symmetry can remove this degeneracy. The value of $H_{\mathrm{BA}}$ for any arbitrary $\Phi_{m}$ (i.e., any linear combination of $\cos m \varphi$ and $\sin m \varphi$ ) for parallel $x y$ planes may be obtained from the $H_{\mathrm{BA}}^{c c}$ and $H_{\mathrm{BA}}^{s s}$ in Table II using a standard formula. ${ }^{32}$ For brevity of graphical presentation the dependence on distance for states with $m \neq 0$ is given in Fig.

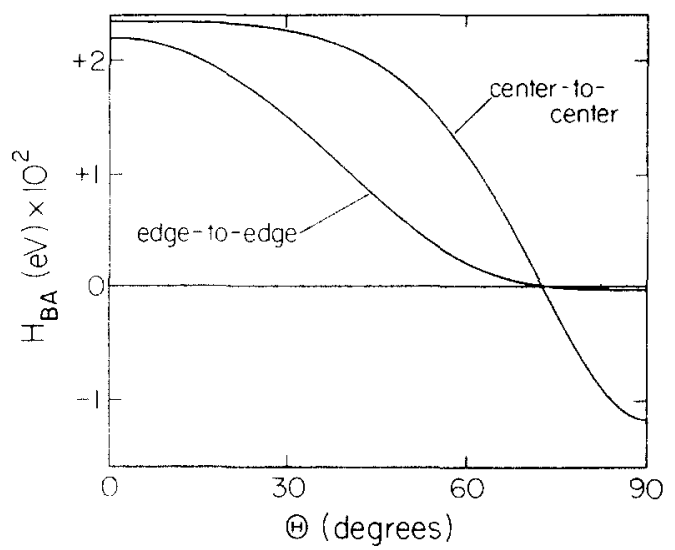

FIG. 11. $H_{\mathrm{BA}}$ as a function of $\Theta$ for $(0, \pi)$ states. $a, b, V_{0}$, and $E$ are as in Fig. 9 for the $(0, \pi)$ states. Shown are results for a constant center-to-center distance of $10 \AA$ and for a constant edge-to-edge distance of $5 \AA$.

12 as a root mean square average of $H_{\mathrm{BA}}$ over $\gamma_{\mathrm{A}}$ and $\gamma_{\mathrm{B}}$, the angles of rotation of $\Psi_{m t u}^{\mathrm{A}}$ and $\Psi_{m t u}^{\mathrm{B}}$ relative to a fixed set of axes located in well $\mathrm{A}$ or well $\mathrm{B}$, respectively, ${ }^{32}$

$$
\begin{aligned}
\left(H_{\mathrm{BA}}\right)_{\mathrm{av}}= & {\left[\frac{1}{4 \pi^{2}} \int_{0}^{2 \pi} \int_{0}^{2 \pi} H_{\mathrm{BA}}^{2}\left(\gamma_{\mathrm{A}}, \gamma_{\mathrm{B}}\right) d \gamma_{\mathrm{A}} d \gamma_{\mathrm{B}}\right]^{1 / 2} } \\
= & \frac{1}{2}\left(\left|H_{\mathrm{BA}}^{c \mathrm{~A}}\right|^{2}+\left|H_{\mathrm{BA}}^{s s}\right|^{2}\right)^{1 / 2} .
\end{aligned}
$$

Nonaveraged $H_{\mathrm{BA}}$ 's are also given in Table I for spheroids in contact.

\section{QUANTIZATION AND SINGLE-SITE WAVE FUNCTIONS}

The method used to obtain the wave functions of Sec. II is described next.

\section{A. Expansion for the separated wave functions}

The functions sought are solutions to Eq. (5), valid both inside and outside the spheroidal well. Neglecting the boundary conditions at $\xi=\xi_{0}$ the wave function inside the well can be separated as in Eq. (7a), yielding

TABLE II. $H_{\mathrm{BA}}$ as a function of center-to-center distance for various $(m, \pi)$ states at $\Theta=0^{\circ}$ and $90^{\circ}$.

\begin{tabular}{ccccc}
\hline \hline State & $R(\AA)$ & $H_{\mathrm{BA}}^{c c, s s}\left(\Theta=0^{\circ}\right)^{\mathrm{b}}$ & $H_{\mathrm{BA}}^{c c}\left(\Theta=90^{\circ}\right)$ & $H_{\mathrm{BA}}^{s s}\left(\Theta=90^{\circ}\right)$ \\
\hline$(1, \pi)$ & 10 & $1.02(-2)$ & $4.16(-2)$ & $-4.78(-3)$ \\
& 15 & $2.69(-4)$ & $6.89(-4)$ & $-6.33(-5)$ \\
& 20 & $9.16(-6)$ & $1.97(-5)$ & $-1.46(-6)$ \\
$(2, \pi)$ & 25 & $3.60(-7)$ & $7.06(-7)$ & $-4.39(-8)$ \\
& 10 & $3.81(-3)$ & $-4.49(-2)$ & $1.76(-2)$ \\
& 15 & $6.08(-5)$ & $-4.76(-4)$ & $1.61(-4)$ \\
& 20 & $1.45(-6)$ & $-1.10(-5)$ & $3.10(-6)$ \\
$(4, \pi)$ & 25 & $4.36(-8)$ & $-3.49(-7)$ & $8.35(-8)$ \\
& 10 & $3.77(-4)$ & $-4.29(-2)$ & $3.68(-2)$ \\
& 15 & $2.02(-6)$ & $-1.29(-4)$ & $1.09(-4)$ \\
& 20 & $2.24(-8)$ & $-1.48(-6)$ & $1.17(-6)$ \\
& 25 & $3.76(-10)$ & $-3.00(-8)$ & $2.19(-8)$ \\
\hline
\end{tabular}

\footnotetext{
"The states and wells used are the same as those in Table I. $H_{\mathrm{BA}}^{s s}$ is $H_{\mathrm{BA}}$ calculated using $\Phi_{m}(\varphi)=\sin m \varphi$ in each well. $H_{\mathrm{BA}}^{c c}$ is for $\Phi_{m}(\varphi)=\cos m \varphi$ in each well. The number in parentheses is the power of 10 to be multiplied by the number preceding it.

${ }^{\mathrm{b}}$ In the $\Theta=0^{\circ}$ orientation $H_{\mathrm{BA}}^{c c}=H_{\mathrm{BA}}^{s s}$.
} 


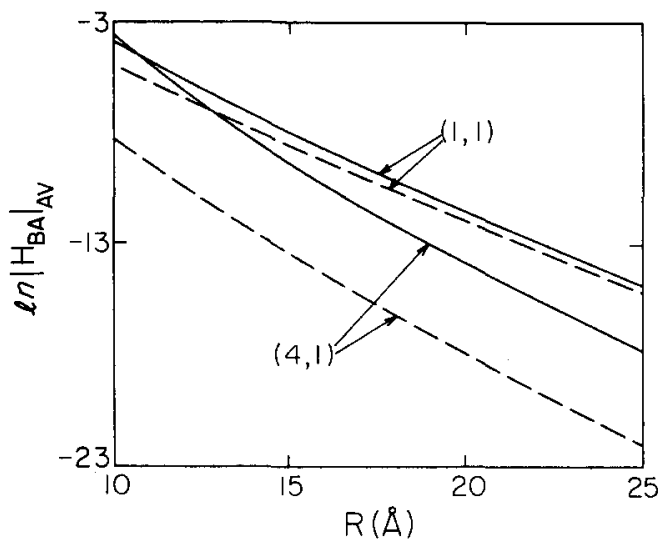

FIG. 12. $\ln \left(H_{\mathrm{BA}}\right)_{\mathrm{av}}$ as a function of center-to-center distance for a pair of $(1, \pi)[=(1,1)]$ states in each well and for a pair of $(4, \pi)[=(4,1)]$ states. In all cases $a, b$, and $E$ are as in Fig. 9. $V_{0}$ for the $(1, \pi)$ states is 7.9296 and for the $(4, \pi)$ states is $17.3530 \mathrm{eV}$. Solid lines are for $\Theta=90^{\circ}$ and dashed lines for $\Theta=0^{\circ}$.

$$
\begin{aligned}
& \frac{d^{2} \Phi_{m}^{i}}{d \varphi^{2}}+m^{2} \Phi_{m}^{i}=0, \\
& \frac{d}{d \eta}\left\{\left(1-\eta^{2}\right) \frac{d S_{m n}^{i}}{d \eta}\right\} \\
& \quad+\left\{\frac{d^{2}}{4} \eta^{2} k_{i}^{2}-\frac{m^{2}}{1-\eta^{2}}+\lambda_{m n}^{i}\right\} S_{m n}^{i}=0, \\
& \frac{d}{d \xi}\left\{\left(1+\xi^{2}\right) \frac{d R_{m n}^{i}}{d \xi}\right\} \\
& \quad+\left\{\frac{d^{2}}{4} \xi^{2} k_{i}^{2}+\frac{m^{2}}{1+\xi^{2}}-\lambda_{m n}^{i}\right\} R_{m n}^{i}=0
\end{aligned}
$$

for the separated equations.

Any choice of $k_{i}^{2}$ (i.e., of energy for fixed $V_{0}$ ) yields a sequence of discrete eigenvalues $\lambda_{m n}{ }_{m n}$. The subscript $m$ describes the eigenvalue in Eq. (12). The subscript $n$ orders the eigenvalues $\lambda_{m n}^{i}$ and was defined in Sec. II. The $\psi_{m n}^{i o o}$ are odd or even with respect to reflection in the $x y$ plane as $n-m$ is odd or even.

Equations (12) through (14) for a given $k_{i}^{2}$ yield a particular set of solutions $\psi_{m n}^{i}$ of the form shown in Eq. (7a). The function $\psi_{m n}^{o}$, neglecting the boundary conditions at $\xi=\xi_{0}$, can similarly be separated as in Eq. (7b). The related separated differential equations are identical to Eqs. (12)-(14), with $i$ replaced by $o$. The angle function $\Phi_{m}(\varphi)$ is the same both inside and outside the well so the superscripts $i$ and $o$ are suppressed in Eq. (7).

The inner and outer radial functions $R_{m n}^{i}\left(\xi ; k_{i}^{2}\right)$ and $R_{m n}^{o}\left(\xi ; k_{o}^{2}\right)$ were evaluated through their expansions in spherical and modified spherical Bessel functions $j_{n}\left(d k_{i} \xi / 2\right)$ and $k_{n}\left(d\left|k_{o}\right| \xi / 2\right)$, respectively. The angular functions $S_{m n}^{i}\left(\eta ; k_{i}^{2}\right)$ and $S_{m n}^{o}\left(\eta ; k_{o}^{2}\right)$ were evaluated through their expansions in the associated Legendre functions $P_{n}^{m}(\eta)$. The radial and angular functions $R_{m n}$ and $S_{m n}$ in Eq. (7), their expansion coefficients, and the eigenvalues $\lambda_{m n}$ are discussed by Flammer, ${ }^{33}$ who presents tables of both. Hodge ${ }^{34}$ has given an algorithm for obtaining them which was easily programmed and was used for the calculations in the present paper. ${ }^{35}$ The radial and angular functions were converged to at least four significant figures in all cases. ${ }^{36}$

\section{B. Quantization in the limit $V_{0} \rightarrow \infty$}

In this case the sum for $\psi$ in Eq. (6) reduces to a single term and the allowed energy levels are those for which $k_{i}^{2}$ yields

$$
R_{m n}^{i}\left(\xi_{0} ; k_{i}^{2}\right)=0
$$

since the wave function must vanish for $\xi \geqslant \xi_{0}$. In the spherical limit $(b \rightarrow a)$ the energy eigenvalues given in Fig. 6(a), are simply those for which $b k_{i}$ is a zero of the $n$ th-order spherical Bessel function. An oblate spheroidal square well has been used as a model for the potential in which a nucleon moves in the nucleus. ${ }^{37}$ In this context the energy levels have been calculated previously in the limit $V_{0}=\infty .^{38}$

\section{Quanitzation for finite $V_{0}$}

The wave functions in this case must be written as a sum of inner or outer functions because both the angular and radial parts of the wave functions depend on the energy. Quantization is accomplished by requiring that the wave function and its normal derivative be continuous at $\xi=\xi_{0}$, i.e.,

$$
\begin{aligned}
& \lim _{\xi \rightarrow \xi_{o}^{-}} \Psi_{m t u}^{i}\left(\xi, \eta, \varphi ; k_{i}^{2}\right)=\lim _{\xi \rightarrow \xi_{o}^{+}} \Psi_{m t u}^{o}\left(\xi, \eta, \varphi ; k_{o}^{2}\right) \\
& \lim _{\xi \rightarrow \xi_{o}^{-}} \frac{\partial \Psi_{m t u}^{i}}{\partial \xi}\left(\xi, \eta, \varphi ; k_{i}^{2}\right)=\lim _{\xi \rightarrow \xi_{o}^{+}} \frac{\partial \Psi_{m t u}^{o}}{\partial \xi}\left(\xi, \eta, \varphi ; k_{o}^{2}\right) .
\end{aligned}
$$

Continuity of $\partial \Psi / \partial \eta$ at the boundary $\xi=\xi_{0}$ is ensured by Eq. (16).

The following method was used to determine the energy eigenvalues $E_{m t u}$ for which Eqs. (16) and (17) are satisfied. Each outer angular function $S_{m n}^{o}\left(\eta ; k_{o}^{2}\right)$ was expanded in the complete set of inner angular functions $S_{m n}^{i}\left(\eta ; k_{i}^{2}\right)$, thereby yielding $\Psi_{m t u}$ as an expansion in $S_{m n}^{i}\left(\eta ; k_{i}^{2}\right)$ for both $\xi \leqslant \xi_{0}$ and for $\xi \geqslant \xi_{0}$. Equating the two expansions term by term at $\xi=\xi_{0}$ yields

$$
\mathbf{C}^{i}=\mathbf{M C} \text {. }
$$

In Eq. (18) $\mathbf{C}^{i}$ denotes the column vector with elements $\left(C_{m+s}^{i}, C_{m+s+2}^{i}, \ldots\right)$ and $C^{o}$ denotes $\left(C_{m+s}^{o}, C_{m+s+2}^{o}, \ldots\right)$, the $C_{n}^{i}$ 's and the $C_{n}^{o}$ 's being the coefficients appearing in Eq. (6). Both vectors are of infinite dimension but are truncated in practice. The elements of the matrix $\mathbf{M}$ depend on the energy and on the quantum number $m$, and are given by

$$
M_{k j}=\frac{\left\langle S_{m q}^{i} \mid S_{m p}^{o}\right\rangle R_{m p}^{o}\left(\xi_{0} ; k_{o}^{2}\right)}{\left\langle S_{m q}^{i} \mid S_{m q}^{i}\right\rangle R_{m q}^{i}\left(\xi_{0} ; k_{i}^{2}\right)},
$$

where

$$
\begin{aligned}
& p=m+2(j-1)+s, \quad j \geqslant 1 ; \\
& q=m+2(k-1)+s, \quad k \geqslant 1 .
\end{aligned}
$$

Similarly, continuity of the normal derivative at $\xi=\xi_{0}$ yields the matrix equation

where

$$
\mathbf{C}^{i}=\mathbf{M}^{\prime} \mathbf{C}^{\circ}
$$

$$
M_{k j}^{\prime}=\frac{\left\langle S_{m q}^{i} \mid S_{m p}^{o}\right\rangle d R_{m p}^{o}\left(\xi_{0} ; k_{o}^{2}\right) / d \xi_{0}}{\left\langle S_{m q}^{i} \mid S_{m q}^{i}\right\rangle d R_{m q}^{i}\left(\xi_{0} ; k_{i}^{2}\right) / d \xi_{0}}
$$

and $p$ and $q$ are again given by Eq. (19b). Equations (18) and (20) yield 


$$
\mathbf{C}^{o}=\mathbf{M}^{-1} \mathbf{M}^{\prime} \mathbf{C}^{o} \text {. }
$$

Thus, $\mathbf{C}^{o}$ is an eigenvector of the matrix $\mathbf{M}^{-1} \mathbf{M}^{\prime}$ having a unit eigenvalue. An energy eigenvalue and eigenvector $\mathbf{C}^{\circ}$ is found by iterating the energy in Eq. (22) to obtain an eigenvector with unit eigenvalue. The inner expansion coefficients $\mathrm{C}^{i}$ are then obtained using Eq. (18) and this $\mathbf{C}^{o}$. The wave functions are normalized by the factor $A_{m t u}$ in Eq. (6), $A_{m t u}$ being $\left.\left\langle\Psi_{m t u}^{u}\right| \Psi_{m t u}^{u}\right)^{-1 / 2}$, where $\Psi_{m t u}^{u}$ is the unnormalized solution.

\section{DISCUSSION}

In this section the dependence of $H_{\mathrm{BA}}$ on distance and the factors affecting the orientation dependence of $H_{\mathrm{BA}}$ are discussed.

Figures 9 and 12 give plots of $\ln \left|H_{\mathrm{BA}}\right|$ or $\ln \left(H_{\mathrm{BA}}\right)_{\mathrm{av}}$ vs distance and it is seen that $H_{\mathrm{BA}}$ decreases, as expected, predominantly exponentially with distance for all the states considered. In Appendix B analytical expressions for $H_{\mathrm{BA}}$ as a function of distance are derived for certain states of spherical wells. ${ }^{39}$ For spherical states analogous to the $(0, \sigma)$ states of spheroidal wells, we find [Eq. (B10)]

$$
H_{\mathrm{BA}}=-\frac{\hbar^{2} A_{0}^{2}}{2 m_{e} \alpha^{2} R} \exp (-\alpha R)
$$

where $A_{0}$ is the radial normalization constant, given in Appendix $B$, for single-well wave functions. $R$ is the distance between the well centers, and $\alpha=\left(-2 m_{e} E / \hbar^{2}\right)^{1 / 2}$. It can be seen explicitly that the large- $R$ asymptotic distance dependence of $\ln \left|H_{\mathrm{BA}}\right|$ is linear.

For spherical states analogous to the spheroidal $(0, \pi)$ states one has [Eqs. (B11) and (B12)]

$$
H_{\mathrm{BA}}=-\frac{C}{\alpha}\left(\frac{1}{(\alpha R)^{2}}+\frac{1}{(\alpha R)^{3}}\right) \exp (-\alpha R) \quad\left(\Theta=90^{\circ}\right)(
$$

and

$$
H_{\mathrm{BA}}=\frac{C}{\alpha}\left(\frac{1}{\alpha R}+\frac{2}{(\alpha R)^{2}}+\frac{2}{(\alpha R)^{3}}\right) \exp (-\alpha R) \quad\left(\Theta=0^{\circ}\right) .
$$

The constant $C$ is defined by comparing with Eqs. (B11) and (B12) and using Eq. (B3).

In both orientations it is seen that $\ln \left|H_{\mathrm{BA}}\right|$ depends predominantly linearly on distance. The exponential dependence arises from the overlap of the radial part of $\Psi^{\mathrm{A}}$, a modified spherical Bessel function, with $\Psi^{\mathrm{B}}$. Since the outer spheroidal wave functions are composed of sums of modified spherical Bessel functions a similar distance dependence of $H_{\mathrm{BA}}$ is expected and is found.

In general, the functional form of the preexponential part of the distance dependence of $H_{\mathrm{BA}}$ is dependent on the potential functions at the two centers. For one-dimensional square wells $H_{\mathrm{BA}}$ is proportional to an exponential function of $R .^{40}$ For transfer of an electron between two protons $H_{\mathrm{BA}}$ is a polynomial in powers of $R$ multiplied by an exponential. ${ }^{41}$ The dominant part of $H_{\mathrm{BA}}$ in all these cases is a decaying exponential but the potential shape and nodal structure of the wave functions cause slowly varying deviations from purely exponential behavior.

In analyzing the orientation effects exhibited in Figs. 9 to 12 , there are two principal factors to be considered, the well shape and the nodal structure. They are discussed below, initially for fixed center-to-center and then for fixed edge-to-edge distance:

(1) In the $(0, \sigma)$ case (Fig. 9) there are no nodal complications, and the shape of the spheroidal well favors a larger $\left|H_{\mathrm{BA}}\right|$ in the $\Theta=90^{\circ}$ orientation than in the $\Theta=0^{\circ}$ at fixed center-to-center distance.

(2) [The following results are intended to refer only to $(m, \pi)$ states.] For a fixed center-to-center distance, as the number of nodes in the $\varphi$ portion of the wave function increases (i.e., as $m$ increases) the ratio $\left|H_{\mathrm{BA}}\left(\Theta=90^{\circ}\right)\right| /$ $\left|H_{\mathrm{BA}}\left(\Theta=0^{\circ}\right)\right|$ increases (cf. Figs. 9 and 12). The pairs of $(0, \pi)$ states have larger $\left|H_{\mathrm{BA}}\right|$ 's at $\Theta=0^{\circ}$ than at $\Theta=90^{\circ}$, while all other $(m, \pi)$ states have larger $\left|H_{\mathrm{BA}}\right|$ 's at $\Theta=90^{\circ}$. [Cf. Fig. 12 , including the $(2, \pi)$ case of Table II.]

Result (1) is due to the smaller edge-to-edge distance occurring in the $\Theta=90^{\circ}$ configuration at a fixed center-tocenter distance, and illustrates one geometrical shape effect. We have also observed result (2) for $H_{\mathrm{BA}}$ for spherical-well potentials. ${ }^{42}$ To understand these results we consider the form of $H_{\mathrm{BA}}$ in the spherical case (cf. Appendix B). In essence, with increasing $m$ the wave functions tend increasingly rapidly to zero along their $z$ axes, and so the face-to-face configuration becomes decreasingly favored. We have

$$
\begin{aligned}
H_{\mathrm{BA}}= & -\int_{\text {well B }} V_{0}^{\mathrm{B}} \Psi^{\mathrm{B}^{*}} \Psi^{\mathrm{A}} d \tau_{\mathrm{B}} \\
& \propto \int_{\text {well B }} j_{l^{\prime}}\left(\beta_{\mathrm{B}} r_{\mathrm{B}}\right) P_{l^{\prime}}^{m^{\prime}}\left(\cos \theta_{\mathrm{B}}\right) \Phi_{m^{\prime}}^{*}\left(\varphi_{\mathrm{B}}\right) \\
& \times V_{0}^{\mathrm{B}} k_{l}\left(\beta_{\mathrm{A}} r_{\mathrm{A}}\right) P_{l}^{m}\left(\cos \theta_{\mathrm{A}}\right) \Phi_{m}\left(\varphi_{\mathrm{A}}\right) d \tau_{\mathrm{B}},
\end{aligned}
$$

where the subscripts $A$ and $B$ denote variables appropriate to the functions at site A and B, respectively. $l$ is the total angular momentum quantum number. Spherical states which have similar nodal structures to the $(m, \pi)$ spheroidal states considered have $l=m+1$. The variables $\left(r_{\mathrm{A}}, \theta_{\mathrm{A}}, \varphi_{\mathrm{A}}\right)$ depend implicitly on $\left(r_{B}, \theta_{B}, \varphi_{B}\right)$. Since the integration is over well $\mathrm{B}$ the predominant angular dependence of $H_{\mathrm{BA}}$ on $\Theta$ for the orientations examined in this paper ( $x y$ planes of each well parallel) arises from the function $P_{l}^{m}\left(\cos \theta_{\mathrm{A}}\right)$ which is of the form:

$$
P_{l}^{m}\left(\cos \theta_{\mathrm{A}}\right) \propto \sin ^{m} \theta_{\mathrm{A}} \cos \theta_{\mathrm{A}}, \quad l=m+1, \quad m \geqslant 0 .
$$

In the $\Theta=0^{\circ}$ orientation, the relevant $\theta_{\mathrm{A}}$ approach zero as $R \rightarrow \infty$, for all values of $r_{\mathrm{B}}, \theta_{\mathrm{B}}$, and $\varphi_{\mathrm{B}}$ in well $\mathrm{B}$, and so $\sin ^{m} \theta_{\mathrm{A}}$ goes to zero increasingly rapidly with increasing $m$ in the vicinity of well $\mathrm{B}$. In the $\Theta=90^{\circ}$ orientation, $\theta_{\mathrm{A}}$ approaches $90^{\circ}$ as $R \rightarrow \infty$ and $\cos \theta_{\mathrm{A}}$ tends to zero in the vicinity of well $\mathrm{B}$, but for all $(m, l)$ spherical states considered $\cos \theta_{\mathrm{A}}$ is always raised to the first power. Therefore, as $m$ increases the $\Theta=90^{\circ}$ orientation is increasingly favored over the $\Theta=0^{\circ}$ orientation. For the $(m, l)=(0,1)$ state, only the $\cos \theta_{\mathrm{A}}$ term occurs, and so the $\Theta=0^{\circ}$ configuration is favored. Since the spheroidal wave functions are composed of sums of Legendre polynomials $P_{l}^{m}$, dominated by a few of them, and because of the correspondence between $\eta$ and $\cos \theta$, this explanation is the anticipated one for this orientation dependence in the spheroidal case. Results (1) and (2) are thus at least qualitatively explained. 
TABLE III. Normalized projections $P_{n}^{2}$ of the $(m, t, u)=(0,1,2)$ state in the limit $r \rightarrow \infty$.

\begin{tabular}{cccc}
\hline \hline$n$ & $e=0.1$ & $e=0.5$ & $e=0.9$ \\
\hline 0 & 0.0003 & 0.2153 & 0.4045 \\
2 & 0.9997 & 0.7825 & 0.5637 \\
4 & 0.0000 & 0.0022 & 0.0315 \\
6 & 0.0000 & 0.0000 & 0.0004 \\
\hline
\end{tabular}

"The quantity $P^{2}$ is defined in Eq. (28); $e$ is the eccentricity of the well at a constant volume of $251.25 \AA^{3}$. For these calculations, $V_{0}=10 \mathrm{eV}$. Because $n-m$ is even, $P_{n}^{2} \equiv 0$ for all odd $n$. The eccentricity of the wells in Figs. $3-5$ and $9-12$ is $\sim 0.85$.

Although distances in experiments are often quoted as center-to-center distances it is useful, because of the exponential decay of the wave functions, to examine the results at constant edge-to-edge distances. The data presented are sufficient to make comparisons of $H_{\mathrm{BA}}$ for fixed edge-to-edge separation. Equal edge-to-edge separations in the two orientations are obtained by comparing $H_{\mathrm{BA}}$ for which the centerto-center separation is $4.6 \AA[=2(a-b)]$ larger in the $\Theta=90^{\circ}$ orientation. For the systems examined in this paper, $H_{\mathrm{BA}}$ was always larger at $\Theta=0^{\circ}$ than at $\Theta=90^{\circ}$ for $m<4$ and was roughly equal for $m=4$ at the two $\Theta$ 's.

The explanation presumably lies in a geometrical shape effect: In the $\Theta=0^{\circ}$ configuration the spheroids present a larger cross section and smaller thickness to each other, thereby favoring a higher overlap. However, the difference in $\left|H_{\mathrm{BA}}\right|$ 's decreases with increasing $m$ for the reason discussed above, and still larger $m$ 's may reverse the favored $\Theta=0^{\circ}$ result.

While we have largely considered the orientations $\Theta=90^{\circ}$ and $\Theta=0^{\circ}$ in this article for purposes of illustration, other orientations are also of considerable interest. In fact, as $m$ increases, maxima will occur in $H_{\mathrm{BA}}$ at $\Theta$ 's other than $\Theta=0^{\circ}$ [cf. $(2, \pi)$ and $(4, \pi)$ results in Table I]. The angles at which these maxima occur are near the maxima in the angular function $P_{l}^{m}(\cos \theta)$ of the spherical state which has similar nodal structure to the spheroidal state in question $\left[\sim 55^{\circ}\right.$ for the $(2, \pi)$ states and $\sim 63^{\circ}$ for the $(4, \pi)$ states]. It can be seen in Fig. 6 that $\theta_{\mathrm{A}}$ equals $\Theta$ and $\theta_{\mathrm{B}}$ equals $\pi-\Theta$, where $\theta_{\mathrm{A}}$ and $\theta_{\mathrm{B}}$ are the spherical polar angles in each well, so the maxima of $H_{\mathrm{BA}}$ as a function of $\Theta$ are related to maxima in $P_{l}^{m}\left(\cos \theta_{\mathrm{A}}\right)$ and $P_{l}^{m}\left(\cos \theta_{\mathrm{B}}\right)$. This analogy with spherical functions is adequate for the reasons stated previously.

We have also examined the angular dependence of the spheroidal wave functions at $R=\infty$. At a large radial distance each of the outer radial functions has the same asymptotic form, independently of $m$ or $n$ :

$$
R_{m n}^{o}\left(\xi ; k_{o}^{2}\right) \sim \frac{1}{\alpha r} e^{-\alpha r}, \quad r \rightarrow \infty,
$$

where $\alpha \equiv\left|k_{0}\right|=\left(2 m_{e}|E| / \hbar^{2}\right)^{1 / 2}$. Hence, the wave function $\Psi_{m t u}$ at a fixed large $r$ and fixed $\varphi$ varies as

$$
\Psi_{m t u} \sim\left[\sum_{n=0}^{\infty} C_{n}^{o} S_{m n}\left(\eta ; k_{o}^{2}\right)\right] e^{-\alpha r / \alpha r} \quad(r \rightarrow \infty),
$$

where in Eq. (27) we set $\eta=\cos \theta$.

To exhibit the angular dependence of $\Psi_{m t u}$ at large $r$, $\Psi_{m t u}$ in Eq. (27) was projected onto the associated Legendre polynomials $P_{n}^{m}(\cos \theta)$. If the angular probability distribution at large $r$ were insensitive to the nonzero eccentricity of the spheroidal well one would find $\left|\left\langle P_{n}^{m} \mid \Psi_{m t u}\right\rangle\right|^{2}$ equal to zero except for a single value of $n$. Calculated projections, defined as

$$
P_{n}^{2} \equiv \frac{\left|\left\langle\Psi_{m t u} \mid P_{n}^{m}\right\rangle\right|^{2}}{\left|\left\langle P_{n}^{m} \mid P_{n}^{m}\right\rangle\right|^{2}} / \sum_{k} \frac{\left|\left\langle\Psi_{m t u} \mid P_{k}^{m}\right\rangle\right|^{2}}{\left|\left\langle P_{k}^{m} \mid P_{k}^{m}\right\rangle\right|^{2}}, \quad r=\infty,
$$

are given in Table III for wells of three eccentricities, all with a volume of $251.25 \AA^{3}$. The data clearly indicate that even at $r=\infty$ the electron "sees" the nonsphericity of the potential well.

\section{CONCLUSION}

A model electron transfer system involving nonspherical (oblate spheroidal) donor and acceptor sites and a transferable electron has been presented. The wave functions for the isolated donor and acceptor sites and the matrix element for electron transfer have been described and the results of several calculations presented. Thus, a machinery has been developed for the calculation of orientation effects, especially for electron transfer between large aromatic molecules.

The sample calculations illustrate the effects of well shape and nodal structure on the orientation and distance dependence of the electron transfer matrix element. They indicate to a first approximation for the system and states studied, that the geometrical shape effect, for a constant edge-to-edge distance, causes $\left|H_{\mathrm{BA}}\right|$ to be larger for $\Theta=0^{\circ}$ (face-to-face configuration) than for $\Theta=90^{\circ}$ (end-to-end arrangement). This effect is reduced with increasing $m$, a result explained by examining the long-range behavior of a pair of spherical wells. This increasing $m$ effect is expected to apply to states similar to the HOMO or LUMO of large aromatic molecules.

When the results are presented instead at a given center-to-center separation they are significantly influenced by the greater edge-to-edge distance for the $\Theta=0^{\circ}$ configuration (face-to-face), so that now $\left|H_{\mathrm{BA}}\right|$ is largest at $\Theta=90^{\circ}$ for most of the states considered.

\section{ACKNOWLEDGMENTS}

It is a pleasure to acknowledge support of this research by the Office of Naval Research. RJC gratefully acknowledges the support of a National Science Foundation Predoc- 
toral fellowship, 1979-1982. The calculations reported in this paper made use of the Dreyfus-NSF theoretical chemistry computer which was funded through grants from the Camille and Henry Dreyfus Foundation, the National Science Foundation, and the Sloan Fund of the California Institute of Technology.

\section{APPENDIX A: GOLDEN RULE RATE EXPRESSION AND MATRIX ELEMENTS}

The rate expression used to characterize electron transfer in this paper is a Golden Rule rate constant obtained by using a Born-Oppenheimer analysis by Holstein ${ }^{43}$ and has been presented in a paper by Kestner et al. ${ }^{10}$ Another presentation of the derivation which corrects some typographical errors there is found in Ref. 44 . The pertinent results are particularized below to the present model.
The rate expression for transfer of an electron from site $A$ to site $B$ when only a single electronic state is assumed on either site may be written in the Golden Rule and Condon approximations as

$$
\begin{aligned}
k_{\mathrm{B}, \mathrm{A}}= & \frac{2 \pi}{\hbar}\left|T_{\mathrm{BA}}\right|^{2}\left(\frac{1}{Q_{A}} \sum_{v_{\mathrm{A}}, v_{\mathrm{B}}} e^{-E_{v_{\mathrm{A}}} / k T}\right. \\
& \left.\times\left.\left|\left\langle v_{\mathrm{B}}\right| v_{\mathrm{A}}\right)\right|^{2} \delta\left(E_{v_{\mathrm{B}}}-E_{v_{\mathrm{A}}}\right)\right) .
\end{aligned}
$$

In Eq. (A1) $v_{\mathrm{A}}$ denotes one of a set of nuclear wave functions appropriate to the electron being localized at site $\mathrm{A}, v_{\mathrm{B}}$ denotes a similar set for the electron localized at site $\mathrm{B}$, and $Q_{\mathrm{A}}$ is the nuclear partition function appropriate to the electron being localized on site $A$. For the case of nonorthogonal electronic basis states, $T_{\mathrm{BA}}$ is equal to

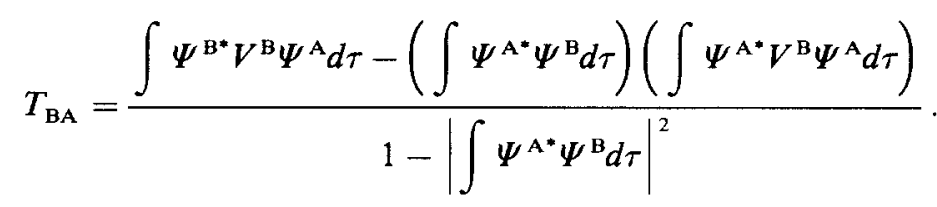

We define

$$
\begin{aligned}
& H_{\mathrm{BA}} \equiv \int \Psi^{\mathrm{B}^{*}} V^{\mathrm{B}} \Psi^{\mathrm{A}} d \tau, \quad S_{\mathrm{AB}}=\int \Psi^{\mathrm{A}^{*}} \Psi^{\mathrm{B}} d \tau,
\end{aligned}
$$

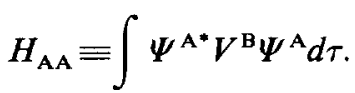

For the type of potential used in this study, the integrals $H_{\mathrm{BA}}$ and $H_{\mathrm{AA}}$ over all space are reduced to integrals over well $\mathrm{B}$, since $V^{\mathrm{B}}$ is zero outside well B. One thus obtains Eq. (9).

\section{APPENDIX B: SPHERICAL WAVE FUNCTIONS AND THEIR ELECTRON TRANSFER MATRIX ELEMENTS}

\section{Spherical wave functions}

In the spherical limit $a \rightarrow b$, the wave function of Eq. (6) assumes the simpler form given in Eq. (B1), where $l$ is the total angular momentum quantum number.

$$
\begin{aligned}
& \Psi_{m l}(r, \theta, \varphi ; E) \\
& \quad=A_{l} N_{m l} \Phi_{m}(\varphi) P_{l}^{m}(\cos \theta) \begin{cases}j_{l}(\beta r) k_{l}(\alpha b) / j_{l}(\beta b), & r \leqslant b, \\
k_{l}(\alpha r), \quad r \geqslant b . & (\mathrm{B} 1)\end{cases}
\end{aligned}
$$

$b$ is the radius of the spherical well, $\beta=\left[2 m_{e}\left(V_{0}+E\right)\right]^{1 / 2} / \hbar, \quad \alpha=\left[-2 m_{e} E\right]^{1 / 2} / \hbar, \quad$ and $\Phi_{m}(\varphi)$ is any linear combination of $\cos m \varphi$ and $\sin m \varphi$ with the absolute square of the coefficients equal to 1 . The angular function $P_{l}^{m}$ is an associated Legendre polynomial. We have used the definition of $P_{l}^{m}$ given by Arfken ${ }^{45}$ The constants $A_{l}$ and $N_{m l}$ are normalization constants for the radial part of the wave function and for the $(m, l)$ spherical harmonic, respectively:

$$
\begin{aligned}
& A_{l}=\left\{\frac{k_{l}^{2}(\alpha b)}{j_{l}^{2}(\beta b)} \int_{0}^{b} j_{l}^{2}(\beta r) r^{2} d r+\int_{b}^{\infty} k_{l}^{2}(\alpha r) r^{2} d r\right\}^{-1 / 2} \\
& N_{m l}=\left\{\frac{2}{2 l+1} \frac{(l+m) !}{(l-m) !} v\right\}^{-1 / 2},
\end{aligned}
$$

where $v$ is $2 \pi$ if $m=0$ and $\pi$ for $m \neq 0$. The $\Psi_{m l}$ given by Eq. (B1) is clearly continuous at the boundary $r=b$. The value of $E$ in $\alpha$ and $\beta$ was determined by making $\partial \Psi_{m l} / \partial r$ continuous there. ${ }^{39}$ To compare the spherical $(m, l)$ states with the spheroidal $(m, \pi)$ states having similar nodal structure we use the relation $l=m+1$. Spherical $(m, l)=(0,0)$ states correspond to the $(0, \sigma)$ states used in Sec. II.

The integrals in Eq. (B2) can be evaluated in closed form to yield

$$
\begin{aligned}
A_{l}= & \left\{k_{l-1}(\alpha b) k_{l+1}(\alpha b)-\left[k_{l}^{2}(\alpha b) / j_{l}^{2}(\beta b)\right]\right. \\
& \left.\times j_{l-1}(\beta b) j_{l+1}(\beta b)\right\}^{-1 / 2}\left(2 / b^{3}\right)^{1 / 2} .
\end{aligned}
$$

\section{Analytical matrix elements for spherical wave functions}

It is possible to transform the matrix element $H_{\mathrm{BA}}$, which is defined as a three-dimensional volume integral in Eq. (A3), to a two-dimensional surface integral. A method due to Bardeen ${ }^{46}$ is used to effect the transformation.

For simplicity, the following discussion is restricted to the special case in which the same wave function is used in each well. That is, both wells have equal radius and depth, and $(m, l)$ is the same for both $\Psi_{m l}^{\mathrm{A}}$ and $\Psi_{m l}^{\mathrm{B}}$. For this case, $H_{\mathrm{BA}}$ is defined as in Eq. (B5),

$$
H_{\mathrm{BA}} \equiv-V_{0} \int_{\text {well B }} \Psi^{\mathrm{A}} \Psi^{\mathrm{B}^{*}} d \tau_{\mathrm{B}} .
$$

In well $\mathrm{B},-V_{0} \Psi^{\mathrm{B}^{*}}$ equals $(E-T) \Psi^{B^{*}}$ and hence Eq. (B5) becomes

$$
H_{\mathrm{BA}}=\int_{\text {well B }} \Psi^{\mathrm{A}}(E-T) \Psi^{\mathrm{B}^{*}} d \tau_{\mathrm{B}}
$$

The subscripts $m l$ on $\Psi_{m l}^{\mathrm{A}}$ and $\Psi_{m l}^{\mathrm{B}}$ have been suppressed. Here $T$ is the kinetic energy operator $-\hbar^{2} \nabla^{2} / 2 m_{e}$. It is assumed in what follows that the centers of the wells lie along the $z$ axis of a right circular cylindrical coordinate system, 
well $\mathrm{B}$ at $z=+R / 2$, and well $\mathrm{A}$ at $z=-R / 2$. The region of integration may be extended beyond the boundary of well B since $(E-T) \Psi^{\mathrm{B}^{*}}$ vanishes outside well B. In particular, the region $z \geqslant 0$, will be used. Also, $\Psi^{\mathrm{A}} E$ equals $T \Psi^{\mathrm{A}}$ in any region that does not include well $\mathrm{A}$, so Eq. (B6) yields

$$
H_{\mathrm{BA}}=\frac{-\hbar^{2}}{2 m_{e}} \int_{z>0}\left(\Psi^{\mathrm{B}^{*}} \nabla^{2} \Psi^{\mathrm{A}}-\Psi^{\mathrm{A}} \nabla^{2} \Psi^{\mathrm{B}^{*}}\right) d \tau .
$$

Gauss' theorem applied to Eq. (B7) yields

$$
\begin{aligned}
H_{\mathrm{BA}}= & \frac{-\hbar^{2}}{2 m_{e}} \int_{\varphi=0}^{2 \pi} \int_{r=0}^{\infty} \\
& \times\left(\Psi^{\mathrm{A}} \frac{\partial \Psi^{\mathrm{B}^{*}}}{\partial z}-\Psi^{\mathrm{B}^{*}} \frac{\partial \Psi^{\mathrm{A}}}{\partial z}\right) r d r d \varphi .
\end{aligned}
$$

Gauss' theorem is applicable to Eq. (B7) because the discontinuity in $\nabla^{2} \Psi^{\mathbf{B}}$ is merely a step discontinuity on the boundary of well $\mathrm{B}$. The integral in Eq. (B8) is written in right circular cylindrical coordinates $(r, \varphi, z)$. The surface of integration is the plane $z=0$, located midway between the two wells.

Equation (B8) can be further simplified by making use of the symmetry of the wave functions. In particular, $\Psi^{\mathrm{A}}$ $=(-1)^{I-m} \Psi^{\mathrm{B}}$ and $\partial \Psi^{\mathrm{A}} / \partial z=(-1)^{I-m+1} \partial \Psi^{\mathrm{B}} / \partial z$ at $z=0$. We have

$$
\begin{aligned}
H_{\mathrm{BA}}= & \frac{\hbar^{2}}{2 m_{e}} \int_{\varphi=0}^{2 \pi} \int_{r=0}^{\infty}(-1)^{l-m} \\
& \times\left(\Psi^{\mathrm{A}} \frac{\partial \Psi^{\mathrm{A}^{*}}}{\partial z}+\Psi^{\mathrm{A}^{*}} \frac{\partial \Psi^{\mathrm{A}}}{\partial z}\right) r d r d \varphi .
\end{aligned}
$$

We have used Eq. (B9) to evaluate $H_{\mathrm{BA}}$ for three particular cases: $(m, l)=(0,0),(0,1)$, and $(1,1)$. Explicit expressions are given in Eqs. (B10)-(B12), wells $B$ and $A$ centered at $z= \pm R / 2$, on the line $x=y=0$,

$$
\begin{aligned}
H_{\mathrm{BA}}= & -\frac{\hbar^{2} A_{0}^{2}}{2 m_{e} \alpha^{2} R} \exp (-\alpha R), \quad m=0, \quad l=0 . \\
H_{\mathrm{BA}}= & \frac{\hbar^{2} 3 A_{1}^{2}}{m_{e} \alpha^{4} R^{3}}\left(1+\alpha R+\frac{\alpha^{2} R^{2}}{2}\right) \\
& \times \exp (-\alpha R), \quad m=0, \quad l=1, \\
H_{\mathrm{BA}}= & -\frac{\hbar^{2} 3 A_{1}^{2}}{2 m_{e} \alpha^{4} R^{3}}(1+\alpha R) \\
& \times \exp (-\alpha R), \quad m=1, \quad l=1 .
\end{aligned}
$$

These choices for $(m, l)$ correspond for $\Theta=0^{\circ}$ to spheroidal states $(0, \sigma),(0, \pi)$, and $(1, \sigma)$, respectively. $(\Theta$ is defined in Fig. 6.) For $\Theta=90^{\circ}$, with parallel $y$ axes, parallel $z$ axes, and superimposed $x$ axes in the two wells, Eq. (B10) corresponds to $(0, \sigma)$ states and Eq. (B12) to $(0, \pi)$ states. For $\Theta=90^{\circ}$ and $(1, \sigma)$ states, Eq. (B11) applies if $\Phi_{m}(\varphi)=\cos m \varphi$, and Eq. (B12) applies if $\Phi_{m}=\sin m \varphi$. It is possible, in principle, to obtain analytical expressions for $H_{\mathrm{BA}}$ for states of higher $m$ values. However, exact numerical results can be easily obtained for spherical wells and it was considered unnecessary to derive exact analytical ones for the present purposes. Approximate analytic ones will be given elsewhere.

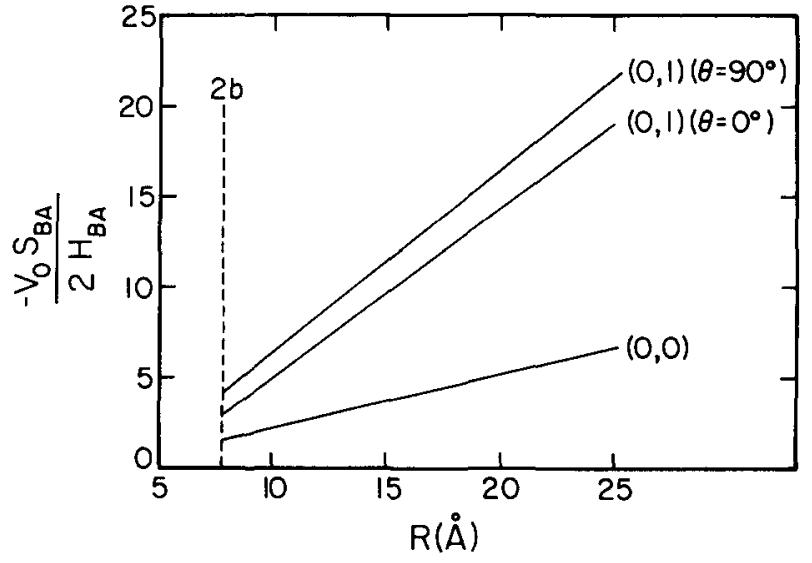

FIG. 13. Dependence of $-2 S_{\mathrm{BA}} / V_{0} H_{\mathrm{BA}}$ for spherical wells on the separation distance $R$ between the well centers. The well radius is $3.9145 \AA$ and $E=-1.1525 \mathrm{eV}$ for all states. The spherical states are labeled as $(m, l)$. $\left[V_{0}=2.48973 \mathrm{eV}\right.$ for $(m, l)=(0,0)$, and $V_{0}=4.19903 \mathrm{eV}$ for $(m, l)=(0,1)$.]

\section{Comparison of electronic matrix elements to overlap integrals for spherical wells}

In an earlier theoretical study of orientation effects ${ }^{19}$ it was assumed that the matrix element $H_{\mathrm{BA}}$ is approximately proportional to the overlap integral $S_{\mathrm{BA}}$. For $(m, l)=(0,0)$ states (i.e., states for which $l=m=0$ with spherical wells of radius $b$ ) the overlap is given by

$$
\begin{aligned}
S_{\mathrm{BA}}(l=0)= & \frac{A_{0}^{2} \exp (-\alpha R)}{2 \alpha^{4} R} \\
& \times\left[\frac{4 \alpha^{2}}{\alpha^{2}+\beta^{2}}+1-e^{-2 \alpha b}+\alpha(R-2 b)\right] .
\end{aligned}
$$

Using Eq. (B10) for $H_{\mathrm{BA}}$, one finds the ratio of $S_{\mathrm{BA}}$ to $H_{\mathrm{BA}}$ is given by

$$
\begin{aligned}
& -\frac{V_{0} S_{\mathrm{BA}}}{2 H_{\mathrm{BA}}}=1+\frac{V_{0}}{4|E|}\left[1-e^{-2 \alpha b}+\alpha(R-2 b)\right], \\
& m=l=0 .
\end{aligned}
$$

For spherical $(m, l)=(0,1)$ states at orientations $\Theta=0^{\circ}$ and $\Theta=90^{\circ}, H_{\mathrm{BA}}$ is given by Eqs. (B11) and (B12). No closed form expressions for $S_{\mathrm{BA}}$ are available, but these overlap integrals are readily evaluated numerically. The results are shown in Fig. 13.

Since $H_{\mathrm{BA}}$ is the overlap of the two functions in well B multiplied by $V_{0}^{\mathrm{B}}$ the quantity plotted would be approximately constant if $S_{\mathrm{BA}}$ were proportional to $H_{\mathrm{BA}}$. It is seen that the ratio grows linearly with distance and depends on orientation. Over short variations of distance proportionality may be an adequate approximation but for large variations it clearly breaks down.

On the basis of the spheroidal results in Table I we again expect $T_{\mathrm{BA}} \approx H_{\mathrm{BA}}$ to within $10 \%$, at least for the results in Fig. 13, for which $R \geqslant 10 \AA$. On this basis, Fig. 13 also represents a plot of the ratio $-V_{0} S_{\mathrm{BA}} / 2 T_{\mathrm{BA}}$.

${ }^{1}$ V. V. Klimov, A. V. Klevanik, V. A. Shuvalov, and A. A. Krasnovsky, FEBS Lett. 82, 183 (1977). 
${ }^{2}$ J. Fajer, M. S. Davis, A. Forman, V. V. Klimov, E. Dolan, and B. Ke, J. Am. Chem. Soc. 102, 7143 (1980).

${ }^{3}$ M. S. Davis, A. Forman, and J. Fajer, Proc. Natl. Acad. Sci. U. S. A. 76, $4170(1979)$.

${ }^{4}$ M. W. Makinen, S. A. Schichman, S. C. Hill, and H. B. Gray, Science 222, 929 (1983).

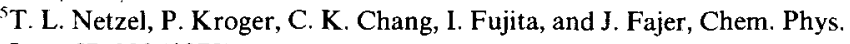
Lett. 67, $223(1979)$.

${ }^{6}$ T. L. Netzel, M. A. Bergkamp, and C. K. Chang, J. Am. Chem. Soc. 104, 1952 (1982).

${ }^{7} \mathrm{G}$. McLendon (private communication).

${ }^{8}$ V. G. Levich and R. R. Dogonadze, Collect. Czech. Chem. Commun. 26, 193 (1961); Translator, O. Boshko, University of Ottawa, Ontario, Canada.

${ }^{9}$ R. R. Dogonadze, A. M. Kuznetsov, and M. A. Vorotyntsev, Phys. Status Solidi B 54, 125, 425 (1972).

${ }^{10}$ N. R. Kestner, J. Logan, and J. Jortner, J. Phys. Chem. 78, 2148 (1974).

"B. S. Brunschwig, J. Logan, M. D. Newton, and N. Sutin, J. Am. Chem. Soc. 102, 5798 (1980).

${ }_{12}^{12}$ P. Siders and R. A. Marcus, J. Am. Chem. Soc. 103, 741 (1981).

${ }^{13}$ K. Ruedenberg and C. W. Scherr, J. Chem. Phys. 21, 1565 (1953); 22, 151 (1954).

${ }^{14}$ (a) O. Schmidt, Z. Phys. Chem. B 39, 59 (1938); (b) 42, 83 (1939); (c) 44, 194 (1939); (d) 47, 1 (1940); O. Schmidt and H. Schmidt, ibid. 44, 185 (1939). ${ }^{15}$ J. R. Platt, J. Chem. Phys. 22, 1448 (1954).

${ }^{16}$ (a) I. V. Alexandrov, R. F. Khairutdinov, and K. I. Zamaraev, Chem. Phys. 32, 123 (1978); J. V. Beitz and J. R. Miller, J. Chem. Phys. 71, 4579 (1979); (b) In Alexandrov et al. [Ref. 16(a)] the electron transfer between the biphenyl anion and napthalene had a $\beta(\equiv 2 \alpha)$ of $1.2 \AA^{-1}$ and that between the same anion and phenylethylene had $\beta=1.0 \AA^{-1}$. In another study [J. R. Miller (private communication)] the $\beta$ for the same anion reacting with acridine was $1.1 \AA^{-1}$. Electron transfer between tetramethylenediamine with a pyrine cation and with a biphenyl cation each had a $\beta$ of $1.15 \AA^{-1}$ [J. R. Miller and J. V. Beitz, J. Chem. Phys. 74, 6746 (1981)]. The $\beta$ inferred from conduction between two metals separated by monolayers of fatty acid salts of varying chain lengths varied between 1.0 and 1.5. [B. Mann and H. Kuhn, J. Appl. Phys. 42, 4398 (1971); M. Sugi, T. Fukui, and S. Iizima, Appl. Phys. Lett. 27, 559 (1975); E. E. Polymeropoulos, J. Appl. Phys. 48, 2404 (1977)]. $\beta$ 's inferred from photoconduction involving excited chromophores separated from an acceptor by a fatty acid layer of known chain length were about 0.3 to 0.5 [M. Sugi, K. Nembach, D. Mobius, and H. Kuhn, Solid State Commun. 15, 1867 (1974); H. Killesreiter and H. Baessler, Chem. Phys. Lett. 11, 411 (1971); H. Kuhn, J. Photochem. 10, 111 (1979)].

${ }^{17}$ Intramolecular exchange mechanisms involving intervening orbitals of atoms (or of molecules) have been examined in the literature [J. Halpern and L. E. Orgel, Discuss. Faraday Soc. 29, 32 (1960); H. M. McConnell, J. Chem. Phys. 35, 508 (1961); S. Larsson, J. Am. Chem. Soc. 103, 4034 (1981); S. Larsson, J. Chem. Soc. Faraday Trans. 2 79, 1375 (1983); S. Larsson, J. Phys. Chem. 88, 1321 (1984); D. N. Beratan and J. J. Hopfield, J. Am. Chem. Soc. 106, 1584 (1984).] In such models the transfer rate varies approximately as a power $1 / x^{n-1}$, where $n$ is the total number of orbitals in the shortest path from donor to acceptor which includes the donor, acceptor, and all intervening atomic or molecular centers, and $x>1$ [cf. S. Larsson, J. Phys. Chem. 88, 1321 (1984); D. N. Beratan and J. J. Hopfield, J. Am. Chem. Soc. 106, 1584 (1984)]. If each orbital occupies a length $a$, such a model yields a rate which is proportional to $\exp \left[-\left(a^{-1} \ln x\right) r\right]$. Thus, these "exchange" models yield an exponential dependence of the rate on $r$. In such theories there will be differences of rate with exchange path, according as the paths are assumed to be composed of bonded species or of solvent molecules.

${ }^{18}$ S. A. Rice and M. J. Pilling, Progr. React. Kinet. 9,93 (1978).

${ }^{19}$ B. Brocklehurst, J. Phys. Chem. 83, 536 (1979).

${ }^{20}$ A. B. Doktorov, R. F. Khairutdinov, and K. I. Zamaraev, Chem. Phys. 61, $351(1981)$.

${ }^{21}$ G. H. Loew, in Iron Porphyrins, Part I, edited by A. B. P. Lever and H. B. Gray (Addison-Wesley, Reading, MA, 1983), p. 1

${ }^{22}$ D. Spangler, G. M. Maggiora, L. L. Shipman, and R. E. Christoffersen, J. Am. Chem. Soc. 99, 7470, 7478 (1977).

${ }^{23}$ A. Dedieu, M.-M Rohmer, and A. Veillard, Adv. Quantum Chem. 16, 43 (1982).

24J. D. Petke and G. M. Maggiora, Chem. Phys. Lett. 97, 231 (1983).

${ }^{25}$ Newton has performed $a b$ initio calculations of the thermal matrix ele- ment for the $\mathrm{Fe}\left(\mathrm{H}_{2} \mathrm{O}\right)_{6}^{3+} / \mathrm{Fe}\left(\mathrm{H}_{2} \mathrm{O}\right)_{6}^{2+}$ self-exchange reaction for $\mathrm{Fe}-\mathrm{Fe}$ separation distances up to $10 \AA$. [cf. M. D. Newton, Int. J. Quantum Chem. Symp. 14, 363 (1980)].

${ }^{26}$ M.-H Whangbo and K. R. Stewart, Isr. J. Chem. 23, 133 (1983).

${ }^{27}$ W. J. Pietro, D. E. Ellis, T. J. Marks, and M. A. Ratner, Mol. Cryst. Liq Cryst. 105, 273 (1984).

${ }^{28}$ See, e.g., J. R. Miller and J. V. Beitz, Ref. 16(b).

${ }^{29}$ In all cases, energies were obtained iteratively and are accurate to not fewer than five digits.

${ }^{30}$ Recent reviews include those of N. Sutin, Progr. Inorg. Chem. 30, 441 (1983); L. Eberson, Adv. Phys. Org. Chem. 18, 79 (1982); R. D. Cannon, Electron Transfer Reactions (Butterworths, London, 1980); D. DeVault, Q. Rev. Biophys. 13, 387 (1980); J, Ulstrup, Charge Transfer Processes in Condensed Media, Lecture Notes in Chemistry, No. 10 (Springer, Berlin, 1979). An early review is given in R. A. Marcus, Annu. Rev. Phys. Chem. 15,155 (1964).

${ }^{31}$ R. T. Morrison and R. N. Boyd, Organic Chemistry, 3rd ed. (Allyn and Bacon, Boston, 1973), p. 968.

${ }^{32}$ Let the $x$ axes of the two wells both be in the plane of Fig. 6. Rotation of an eigenfunction in well $\mathrm{A}, \Psi_{m i u}^{\mathrm{A}}\left(\xi_{\mathrm{A}}, \eta_{\mathrm{A}}\right) \cos m \varphi_{\mathrm{A}}$ by an angle $\gamma_{\mathrm{A}}$ about the fixed $z$ axis yields a rotated function $\Psi_{m e u}^{\mathrm{A}}\left(\xi_{\mathrm{A}}, \eta_{\mathrm{A}}\right) \cos m\left(\varphi_{\mathrm{A}}-\gamma_{\mathrm{A}}\right)$. A rotated $\psi_{m i u}^{\mathrm{B}}\left(\xi_{\mathrm{B}}, \eta_{\mathrm{B}}, \varphi_{\mathrm{B}}\right)$ may be obtained analogously and one obtains for $H_{\mathrm{BA}}\left(\gamma_{\mathrm{A}}, \gamma_{\mathrm{B}}\right)$,

$$
\begin{aligned}
H_{\mathrm{BA}}\left(\gamma_{\mathrm{A}}, \gamma_{\mathrm{B}}\right)=\int & d \tau_{\mathrm{B}} \Psi_{m t u}^{\mathrm{A}}\left(\xi_{\mathrm{A}}, \eta_{\mathrm{A}}\right) \cos m\left(\varphi_{\mathrm{A}}-\gamma_{\mathrm{A}}\right) \\
& \times V_{0}^{\mathrm{B}} \Psi_{m t u}^{\mathrm{B}}\left(\xi_{\mathrm{B}}, \eta_{\mathrm{B}}\right) \cos m\left(\varphi_{\mathrm{B}}-\gamma_{\mathrm{B}}\right) .
\end{aligned}
$$

Introducing the addition formulas for $\cos m\left(\varphi_{A}-\gamma_{A}\right)$ and for $\cos m\left(\varphi_{B}-\gamma_{B}\right)$ into this expression and using $\mathrm{Eq}$. (11) one sees that due to symmetry, only terms involving $\cos m \varphi_{\mathrm{A}} \cos m \varphi_{\mathrm{B}}$ and $\sin m \varphi_{\mathrm{A}} \sin m \varphi_{\mathrm{B}}$ are nonzero. Then, $H_{\mathrm{BA}}\left(\gamma_{\mathrm{A}}, \gamma_{\mathrm{B}}\right)$ becomes [ $H_{\mathrm{BA}}^{c c} \cos m \gamma_{\mathrm{A}} \cos m \gamma_{\mathrm{B}}$ $\left.+H_{\mathrm{BA}}^{\mathrm{ss}} \sin m \gamma_{\mathrm{A}} \sin m \gamma_{\mathrm{B}}\right] .\left(H_{\mathrm{BA}}\right)_{\mathrm{av}}$ is as given in the second half of Eq. (11).

${ }^{33} \mathrm{C}$. Flammer, Spheroidal Wave Functions (Standford University, Stanford, CA, 1957).

${ }^{34}$ D. B. Hodge, J. Math. Phys. 11, 2308 (1970).

${ }^{35}$ The eigenvalues $\lambda \lambda_{m, n}^{i, o}$ were obtained using Hodge's algorithm, trivially modified as follows: The set $\Lambda=\left\{\lambda_{m n} ; n=m+s, m+s+2, \ldots\right\}$ contains the eigenvalues of a matrix that is, in principle, of infinite dimension. Hodge (Ref. 34) presents a prescription for constructing the elements of the matrix so that the matrix eigenvalues are the eigenvalues of Eqs. (13) and (14). In practice, the matrix must be truncated. The eigenvalues of the truncated matrix are not, in general, equal to the eigenvalues $\lambda_{m n}$ of the differential equations. In order to obtain a finite set $\Lambda_{N}$ $=\left\{\lambda_{m n} ; n=m+s, m+s+2, \ldots, m+2 N+s-2\right\}$ of true eigenvalues (i.e., eigenvalues of the differential equations), a larger set $A_{N}$, of approximate eigenvalues $\lambda_{m n}$ was constructed, where $N^{\prime}>N$ and $\lambda_{m n}$ is an eigenvalue of the $N^{\prime} \times N^{\prime}$ truncated matrix. $N^{\prime}$ was then increased until every element of the subset $\Lambda_{N} \subset A_{N}$, was constant to 11 significant digits upon successive increases of $N^{\prime}$. At that point it was assumed that the first $N$ true eigenvalues $\lambda_{m n}$ had been obtained.

${ }^{36}$ In cases when the expansions did not yield satisfactory convergence the functions were obtained by numerical integration of the appropriate differential equations, for example, Eq. (13) for $S_{m n}^{i}$.

${ }^{37}$ J. Rainwater, Phys. Rev. 79, 432 (1950).

${ }^{38}$ S. Granger and R. D. Spence, Phys. Rev. 83, 460 (1951).

${ }^{39}$ For spherical wells, the method of solution for the single-site wave functions may be found in L. I. Schiff, Quantum Mechanics, 3rd ed. (McGrawHill, New York, 1968), pp. 83-88.

${ }^{40} M$. Redi and J. J. Hopfield, J. Chem. Phys. 72, 6651 (1980).

${ }^{41}$ J. C. Slater, Quantum Theory of Matter, 2nd ed. (McGraw-Hill, New York, 1968), p. 417.

${ }^{42}$ R. J. Cave, P. D. Siders, and R. A. Marcus (to be published).

${ }^{43}$ (a) T. Holstein, Ann. Phys. (N. Y.) 8, 325 (1959); (b) 8, 343 (1959).

${ }^{44}$ P. D. Siders, Ph.D. thesis, California Institute of Technology, Pasadena, California, 1983, Chap. 5, Appendix A. Available from University Microfilms, Ann Arbor, MI, No. 83-22, 674.

${ }^{45} \mathrm{G}$. Arfken, Mathematical Methods for Physicists, 2nd ed. (Academic, New York, 1970), Chap. 12, p. 559.

${ }^{46}$ J. R. Bardeen, Phys. Rev. Lett. 6, 57 (1961). 